\title{
Smart tattoo: technology for monitoring blood glucose in the future
}

\author{
Danny Meetoo, Louise Wong and Bertha Ochieng
}

\begin{abstract}
New ways of measuring blood glucose bring hope of easing the burden of diabetes management for patients living with the conditions. The smart tattoo is an innovation that represents a nascent nanotechnology, which is designed to be implanted within the skin to provide continuous and reliable glucose detection for individuals diagnosed with diabetes mellitus. The potential benefits of the smart tatto are compelling not only due to the potential of these nanodevices to prevent diabetic complications and decrease the related social costs, but also due to ease of use and relative user comfort. However, despite the advantages of the smart tattoo, it is important that health professionals, in embracing nanotechnology, understand the ethical implications of using these innovative devices.
\end{abstract}

Key words: Diabetes mellitus $\square$ Blood glucose monitoring $\square$ Nanomedicine - Nanotechnology a Smart tattoo a Nanomaterials a Ethics

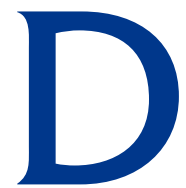

iabetes mellitus is a lifelong metabolic disorder characterised by a chronically elevated reduced [AQ1 isn't it a lack of insulin secretion?] blood insulin secretion, [impaired] insulin action or both (Kumar et al, 2003 [AQ2 v old ref please update]). The prevalence of this condition is escalating at an unprecedented rate across the world without sign of abatement In the UK, for example, an estimated 3.7 million people are living with a diagnosis of diabetes, an increase of 100000 since 2017 (Diabetes UK, 2017 [AQ3 should this be 2018, as I don't think they get the figures that quickly!]). The global picture is equally worrying, with 425 million currently estimated to have diabetes, with the figure expected to peak at 629 million in 2045. If longevity is taken into account, the figure could reach 693 million (International Diabetes Federation, 2017).

In an attempt to halt the problem, health professionals must be given effective training on diabetes prevention and care, in order to reduce the devastating human, social and economic

Danny Meetoo, Programme Leader, MSc Diabetes Care, University of Salford, Rochdale,

D.Meetoo@salford.ac.uk

Louise Wong, job title and department? [AQ4a please provide details] Salford Royal Foundation NHS Trust

Bertha Ochieng, job title and department? [AQ4b please provide details], De Montfort University

Accepted for publication: December 2018 impact of the condition (Meetoo, 2013).

\section{Diabetes management}

People with diabetes constantly walk a tightrope between strict glycaemic control to delay and/or slow the progression of micro- and macrovascular complications. One of the core components of self-care management therefore requires people with type 1 diabetes mellitus (T1DM) and those with type 2 diabetes mellitus (T2DM) treated with insulin to assess glycaemic variability by self-monitoring blood glucose (SMBG) levels (Holt, 2014; Eborall et al, 2015; National Institute for Health and Care Excellence (NICE), 2015). Evidence from several longitudinal landmark studies have conclusively demonstrated that improved glycaemic control reduces the risk of diabetesrelated complications in T1DM (Nathan et al, 1993) and T2DM (UK Prospective Diabetes Trial, 1998; Gerstein et al, 2008; Simon et al, 2008; Holman et al, 2008) and the Kumamoto study (Ohkubo et al, 1995 [AQ5 are there no recent studies?]).

To prevent vascular events, contemporary diabetes management places heavy emphasis on individuals to manage their condition by undertaking a number of behavioural activities, such as healthy eating, taking medication, healthy coping, physical exercise, problem solving, blood pressure and blood glucose monitoring (Meetoo, 2013). These diabetes selfcare behaviours are frequently contingent with the results of SMBG (Tomsky et al, 2008), which is undertaken through a variety of means, dependent on parameters that are individual to each person with diabetes (Poolsup et al, 2009). Unlike $\mathrm{HbA1c}$, which reflects blood glucose levels over the preceding three months, SMBG provides immediate information on a person's glycaemic level, thereby acting as an important guide for adjusting all factors that affect glycaemic control on a more timely basis (Barnett et al, 2008).

\section{Blood glucose monitoring}

Currently, people with T1DM and those managed with sulphonylureas measure their blood glucose by intermittent finger-prick capillary blood sampling. The blood is then placed on a sensor test strip that is read by a handheld electronic reader that uses screen-printed electrodes to measure blood glucose concentration (Wang, 2008). This provides rapid and accurate measurements of blood glucose without the need for laboratory analysis.

However, there are limitations. For example, other than being painful, uncomfortable and likely to lead to non-adherence 
(Heinemann, 2008), this procedure cannot be performed when the person is asleep or at those critical times when dangerous fluctuations in blood glucose concentrations between tests are missed [AQ6 meaning? Please rewrite for clarity] (Burge et al, 2008; Pickup et al, 2008). However, while a standard blood glucose monitor for $\mathrm{SMBG}$ provides discrete and highly accurate BG levels, these readings do not predict future blood glucose levels. The taking and testing of finger-prick blood samples is arguably just one unwelcome consequence of having diabetes. This approach currently represents the only practical way for most patients to manage their glucose levels, but it is becoming more apparent that the traditional method of recording blood glucose levels needs to be replaced by a more sophisticated and pain-free technology.

\section{Blood glucose monitoring with nanotechnology}

If the best way to monitor the outcome of an important matter would be through the use of a continuously running security camera rather than with an intermittently functioning high-quality reflex camera, then it would be logical to suggest that the best way to monitor changes in glycaemia would be via a continuous glucose monitor rather than one yielding intermittent blood glucose results (Figure 1). The development of a completely reliable and accurate technology for a minimally or non-invasive and continuous glucose sensing is therefore considered a would-be 'holy grail' of diabetes care (Clarke et al, 2009; Hughes, 2009).

Over the decades, a single, convenient way of enabling people with diabetes to monitor their blood glucose levels has eluded medical science. Recently, however, one promising technology, known as nanotechnology, has brought the prospect within easy reach by enabling the implantation of glucose biosensors, known as 'smart tattoos' in subcutaneous tissue (Brown et al, 2006; Stein et al, 2007 [AQ7 old refs, please update]). Realisation of such a monitor would, according to Klonoff (2007) [AQ8 old ref, please update], serve such purposes as:

- Detecting and predicting hypoglycaemia and hyperglycaemia in real time

- Assessing mean glycaemia

- Determining the amount of glycaemic variability.

\section{Overview of nanotechnology}

It is beyond the scope of this article to present a detailed explanation of nanotechnology, but to put it briefly it is the 'science of the small' (Marchant, 2009). It has been hailed as the 'next industrial revolution' and promises to have a substantial impact on many areas of our lives (National Technology Initiative, 2000; Maynard, 2006 [AQ9 old refs, please update]). The prefix 'nano', which originates from the Greek word nanos, means 'dwarf', represents 1 billionth of a meter $\left(1 \mathrm{~nm}=10^{-9}\right)$, a dimension that is invisible to the naked eye. To gain a sense of I scale, the diameter of the double-helix DNA molecule is $2 \mathrm{~nm}$, that of a human hair about $100000 \mathrm{~nm}$, while a red blood cell is $7000 \mathrm{~nm}$ across. Atoms are smaller than $1 \mathrm{~nm}$ whereas the size of molecules, including some proteins, ranges between $1 \mathrm{~nm}$ and larger [AQ10 how large? this can't be called a range] (Whitesides, 2003) [AQ11 v old refs, please update].

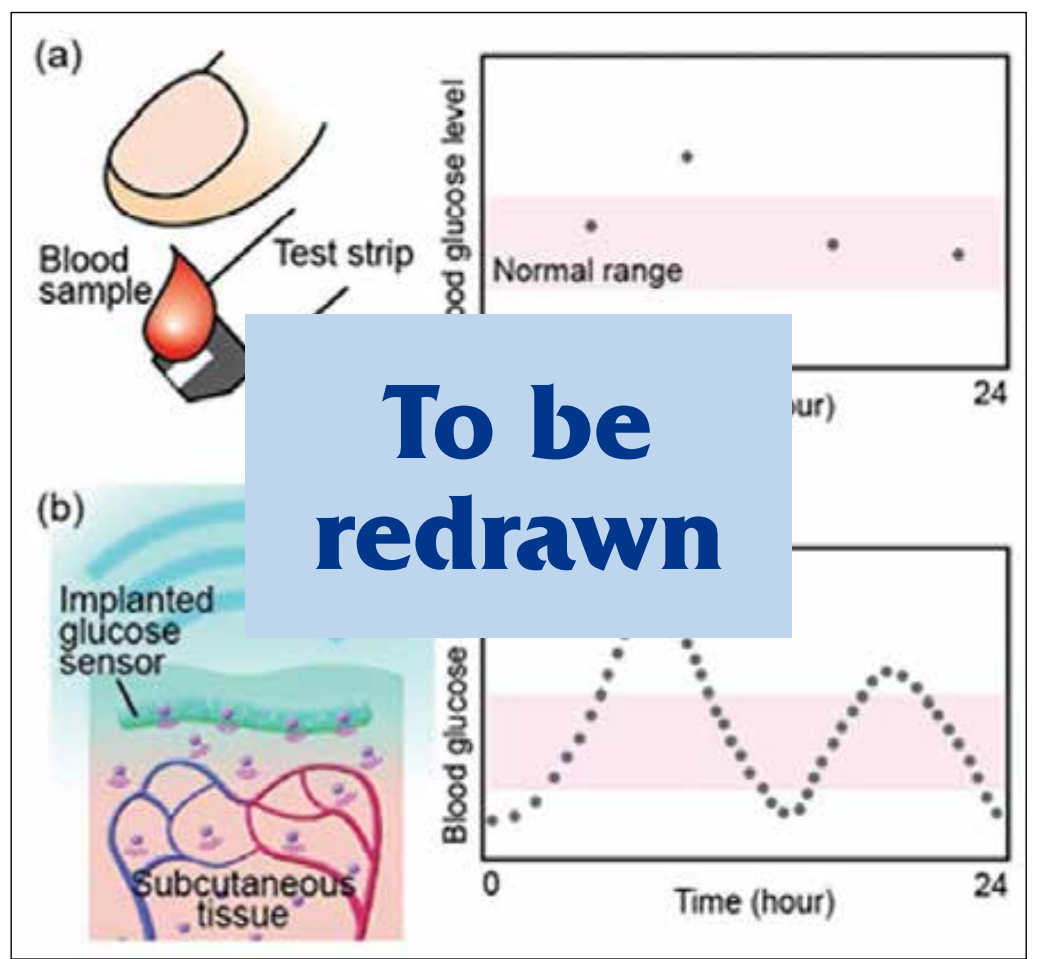

Figure 1. (a) Conventional intermittent glucose monitoring with an enzyme electrode strip (b) Continuous glucose monitoring with smart tattoos (Heo and Takeuchi, 2013)

At this dimension, known as nanoscale, structures and devices often have fundamentally altered properties, including metabolite sensing, controlled porosity, biocompatibility and the ability to target tissues and molecules in the body.

When this science is applied specifically to the problems of medicine, it is referred to as 'nanomedicine' (Freitas, 2005 [AQ12 $\mathrm{v}$ old refs, please update]). The human body has configured many of its biocomponents as nanostructures, including proteins, mitochondria, ion channels, membranes, secretory granules and lysosomes. This has enabled the manufacture of new nanomaterials and structures with application in medicine, ranging from nanoparticles, capsules, films and tubes to complex molecules such as fullerenes (Kroto, 1985). The appeal of nanomedicine therefore stems from the fact that nanoparticles can act as carriers to deliver drugs to targeted cells and tissue sites, including the blood-brain barrier, which are currently not entirely possible [AQ13 It's either possible or not, pls clarify] (Oberdörster et al, 2004 [AQ14 v old ref, please update/delete]; Juliano, 2012).

\section{The smart tattoo}

A simple and convenient way of enabling people with diabetes to monitor their own blood sugar levels has eluded medical science. In recent years, however, continuous glucose monitoring has been available for selected patients via needle-type sensors inserted under the skin. To date, these devices have yet to achieve optimal accuracy and reliability, or widespread use. So the potential benefits of a radical new technique that not 
only eliminates the need for blood samples, but also delivers continuous information are highly desirable. To this end, advances in nanometrology (measurement at nanoscale) are bringing this prospect within reach with the development of smart tattoos, also known as nano tattoos (Stein et al, 2007 [AQ15 old ref, please update]).

The smart tattoo represents a nascent technology designed to be implanted within the skin to provide continuous, reliable glucose detection predominantly for people with T1DM. Its potential benefits are compelling, not only for its ability to prevent diabetes-related complications and reduce related social costs, but also for its ease of use and relative patient comfort. The smart tattoo consists of an intradermally embedded array of biosensors that are exposed to interstitial fluid, enabling the measurement of local changes in glucose, which correlate with blood glucose levels (Caplin et al, 2003 [AQ16 v old ref, please update]). The implant emits visible colour changes corresponding to the glucose levels in the bloodstream, which in turn can be interrogated non-invasively through the skin using a simple optical device (Figure 2) (Long et al, 2008).

For example, the smart tattoo is designed to alert the person when their blood glucose level is high, and insulin is required. The nanomaterials and nanosensors that are part of the smart tattoo have therefore significant advantages because of their small size. They have a high surface area to volume ratio, as well as enhanced optical properties that allow for the improvement of the accuracy and size of the sensors for treatment of diabetes [AQ17 meaning? please rewrite] (Cash and Clark, 2010).

\section{Advantages of smart tattoo}

It may in theory be accepted that just taking one or two blood glucose readings from finger-prick capillaries is sufficient when addressing SMBG. However, evidence has demonstrated how readings are missed when blood glucose is not monitored continuously (Burge et al, 2008; Pickup et al, 2008). This can lead to undesired diabetes consequences, specifically hypoglycaemia (Cash and Clark, 2010). On the other hand, continuous readings empower the individual to successfully prevent and/or manage adverse diabetes events. This can also prove to be advantageous by enabling the individual to have a better understanding of their blood glucose levels at any given point.

The other potential benefits of a smart tattoo are compelling not only for its ability to prevent diabetes-related complications and decrease related social costs, but also for its ease of use and relative comfort. However, unlike regular tattoos, smart tattoos would be only temporary and would be replaced weekly or monthly to account for sensor migration and the loss of signal owing to degradation. This method would eliminate or reduce the need for patients to take blood samples, while allowing data to be collected continuously. It also minimises the chances for infection at the implantation site and avoids other complications of implanted devices, such as capsule formation and the accompanying decreases in glucose transport (Mou et al, 2010).

Arguably, the smart tattoo will make obsolete the current glucose measuring standard of the finger prick, a method widely considered suboptimal in diagnostic efficiency and patient comfort. In the long term, the smart tattoo also has the potential to decrease associated economic costs.

\section{Disadvantages of the smart tattoo}

It is envisaged that the production and application of smart tattoos could prove costly. For this technology to be worthwhile, in this regard a number of factors need be addressed. For example, it is imperative that this technology is tested in realistic, clinical samples even for proof-of-concept design (Cash and Clark, 2010). Second, the cost of smart tattoos needs to be justified by comparing them with other commercially available nanosensors. Finally, to have an impact on diabetes, smart tattoos must demonstrate a substantial advantage over current methods through significant improvements in accuracy without incurring additional costs.

The emerging trends in medical technology mean that the smart tattoo will be a welcome addition to the available range of blood glucose meters. The Freestyle Libre, a flash glucose monitoring system currently available on the NHS across the four UK countries after the individual has met certain criteria is perhaps the closest link to the smart tattoo. In the case of drivers, although interstitial blood glucose values are not an acceptable form of evidence for safe driving, this pain-free technology will arguably be welcomed by people whose diabetes experience is underpinned by blood glucose monitoring. Furthermore, such a meter will provide people with diabetes with a sense of empowerment, control and motivation in understanding and managing their condition (Stephens, 2014).

\section{Controversy and clinical benefits}

The cost benefit of technologies is an important aspect of care. However, health professionals need to be aware that there will always be some variation in results among studies. This may range from differences in patient populations to the subtleties of individual studies' experimental design and protocols, and variability in how the protocol was implemented. For example, factors such as the Hawthorne effect (which describes the change in behaviour when people are being observed), choice of endpoints and random statistical fluctuations can all contribute to the heterogeneity of results.

It is noteworthy that perfect adherence to a self-care regimen

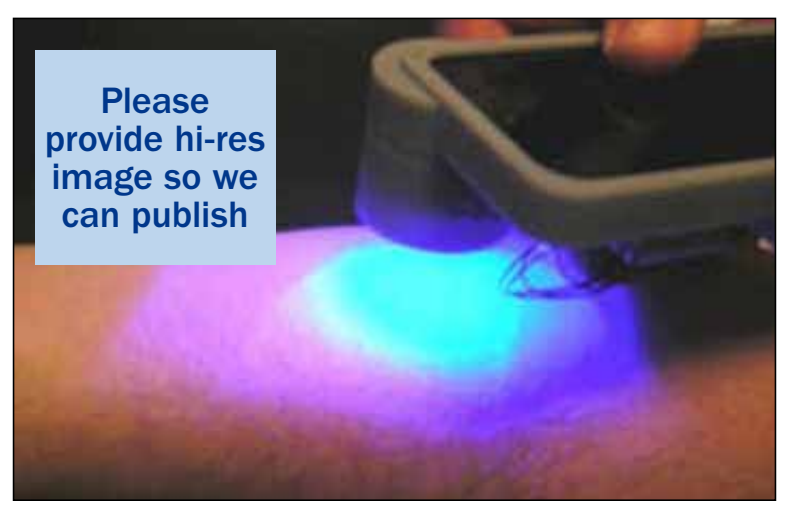

Figure 2. Non-invasive optical instrumentation for measuring blood glucose 
cannot be expected. In many types of studies that involve dosage titration of pharmaceutical agents, one does not expect any differences in HbA1c. Rather, one needs to consider HbA1c and the risk of hypoglycaemia simultaneously (Rodbard, 2015; Vigersky, 2015). When evaluating a CGM [AQ18 computer generated model?] technology, the question should not be: 'Did the use of smart tattoo (or other similar technology such as CGM) result in a change in HbA1c?', nor should it be: 'Did the use of smart tattoo result in a favourable change in the risk of hypoglycaemia?' Instead, the question posed should be: 'Did the introduction of the smart tattoo result in a change in the relationship between risk of hypoglycaemia and HbA1c achieved?' (Rodbard, 2015;Vigersky, 2015).

\section{Smart tattoos and implications for nursing}

Nursing prides itself on being at the patient's side in times of need, whether in hospital or in the community, and the social contract is based on a relationship for providing holistic care throughout the care continuum (Meetoo and Lappin, 2009). Nanotechnology, other than having the potential to redefine health and approaches to care delivery, promises a transition from treating diseases across populations to a person-centred approach focused on 'personalised medicine' (Vlasses and Smeltzer, 2007 [AQ19 old ref, please update]). For example, implants of nanotransmitters and nanosensors would allow individuals to access data transmitted from biochips monitoring such familial diseases as primary hypercholesterolaemia. This is particularly important in allowing affected individuals to take appropriate action to prevent the development of diabetes or diabetes-related diseases.

In the case of people with diabetes, for example, a hyperglycaemic event could send a signal to the care team to prompt initialisation of a customised treatment plan, while nanoparticles could be programmed to administer insulin without a clinician's direct intervention. In time, nanotechnology will increasingly become a diagnostic and therapeutic enabler for the diabetes healthcare team and other health professionals, as well as helping people with diabetes to take more ownership of their condition. The continued development of personalised medicine will not only need a time commitment from carers, but will also require a paradigm shift from consumers as patients to consumers as partners in the decision-making process (Ullman-Cullere et al, 2007 [AQ20 old ref, please update]).This kind of personalised medicine will mean that nurses will need to be knowledgeable about the potential adverse effects of nanotechnology, an area known as nanotoxicology. The aim of this scientific discipline is to determine the extent to which the toxic properties of nanomaterials threaten human beings (Guadagnini et al, 2015) and the environment (Malysheva et al, 2015). Similarly, serious consideration will need to be given, for example, to the prevention of accidental ingestion of nanomaterials during treatment delivery.

\section{Education}

Over-reliance on nanotechnology devices could lead health professionals to assume that they are receiving all the data when in fact this may not be the case (Lewis, 2001 [AQ21 v old ref, please update]). Technological dependence could reduce interprofessional communication, while education for students and trained practitioners through curricular design will be essential to ensure that staff are knowledgeable about the principles underpinning the safe use of nanomaterials. Furthermore, a model of care that includes the provision of round-the-clock nanotechnology support for consumers and nurses will need to be devised and implemented.

Disruptive innovations, such as nanotechnology in health, will inevitably transform how nursing is taught (Kelly, 2016). The use of nanomaterials in nursing, for example, could be the impetus for curricular modifications to educate students and practitioners about the application, control and safe uses of nanomedicines. The continuous emergence of technological innovations may well result in more empowered healthcare consumers at the centre of a well-informed and knowledgeable care team. In achieving this objective, innovative teaching strategies will need to be embraced by nurse educators, who must also be prepared for the teacher-learner relationship to alter in line with the changing needs of the workforce. In additon, the key nursing philosophies of problem-based learning, evidence-based practice and patient-centred care will need to be revisited in the context of technology if they are to remain at the core of education.

Nurses will need to become even more responsible for engaging and driving their own learning journey in an heutagogical approach (Hase and Kenyon, 2001). For ongoing development nurse leaders and educators need to make use of social media platforms to facilitate discussion among their fellow health professionals and also improve the translation of evidence into practice (Moorley and Chinn, 2016). To be ready for this seismic shift nurse educators must therefore have an open-mind set to emerging methods and modalities of learning and cultivate a lifelong learning culture. Embracing the process of learning and unlearning both at organisational and individual level will be essential.

\section{Ethical considerations}

The implementation of nanotechnology will inevitably have ethical implications. Some hold the view that there will be a need to develop new ethical guidelines (Thompson, 2007 [AQ22 $\mathrm{v}$ old ref, please update]), but others contest this (Mnyusiwalla et al, 2003 [AQ23 v old ref, please update]). Given the unknown future design of nanotechnology, Ebbesen et al (2006a; 2006b) [AQ24 old refs, please update] are nevertheless confident that the open-ended nature of Beauchamp and Childress's (2001) principalist model (autonomy, beneficence, nonmaleficence and justice) is sufficiently sensitive to address emerging ethical issues in nanotechnology.

It will be equally important for nanotechnology nurses to be actively involved in contributing to policy-making decisions as well as assisting patients in understanding and consenting to the use of their data, protecting privacy and ensuring that there are mechanisms that allow them to withdraw from participation. Ultimately, however, everyone is likely to require an advocate for the safe and ethical use of nanomaterials in such areas as medical care, the environment, industrial health and the fiscal allocation of research (Traynor, 2006 [AQ25 v old ref, please 
update]). nanotechnology, therefore, has the potential to drive down the costs of care and to create healthy communities that need to use less, rather than more, health care.

Technological dependence could reduce interprofessional communications, and education for students and trained practitioners through curricular design will be essential to ensure that staff are knowledgeable about the principles underpinning the safe use of nanomaterials. Furthermore, a model of care providing round-the-clock nanotechnology support for consumers and nurses will need to be devised and implemented. The advent of nanotechnology will have new implications for clinicians. The concept of routine care could disappear in a world where everything is likely to be customised for individuals presenting with strange or unusual symptoms because the software or minute circuits of the nanomaterials have become infected with viruses (Meetoo and Lappin, 2009).

\section{Conclusion}

This article attempted to review smart tattoo as one of the key technologies most likely to be available in the near future for real time continuous blood glucose monitoring. Arguably, DM can be viewed as a condition of numbers where those affected are asked to monitor their blood glucose levels, insulin doses, exercise, calories, blood pressure and severity of symptoms on numerical scales. With so many numbers from so many self-care activities to keep track of, people with diabetes can benefit from monitoring devices that measure, display, transmit, store and analyse real time data (Kerr, 2010). Smart tattoo seems to be one such technology that promises to change the monitoring of blood glucose.

In today's health care, Morrison (2000:203 [AQ26 v old ref, please update]) writes that 'organ and body functions are restored while the whole patient is ignored.The system is failing to serve'. In the context of technology and to avoid Morrison's prophetic statement, it is imperative that health professionals embrace nanotechnology which promises to revolutionise medicine in the 21st century by eradicating diseases and improving the quality of people's life. The smart tattoo represents a significant and revolutionary technological advancement in monitoring blood glucose and maintaining it within normal parameters to prevent micro- and macrovascular complications.

Continued technological progress, as well as enabling health professionals to develop new and innovative approach to dealing with diabetes, has the potential to decrease the burden of diabetes management on the patients themselves. Furthermore, such progress will continue to improve patient care and its delivery and may one day lead to fully automated treatment systems for people with this condition. It is to be hoped that it is just a matter of time before the management of diabetes is overhauled, thus reversing the current concept of non-adherence as an exception rather than the norm. While embracing nanotechnology, it is equally important for health professionals not to ignore the ethical implications of the smart tattoo from its inception through ongoing research and development to implementation. BJN

Conflict of interest: None
Barnett AH, Krentz AJ, Strojek K et al.The efficacy of self-monitoring of blood glucose in the management of patients with type 2 diabetes treated with a gliclazide modified release-based regimen. A multicentre, randomized, parallel-group, 6-month evaluation (DINAMIC 1 study). Diabetes Obes Metab. 2008; 10(12):1239-47. https://doi.org/10.1111/ .1463-1326.2008.00894.x

--Beauchamp T, Childress J. (2001) Principles of Biomedical Ethics. 5th Edition. Oxford University Press, New York [AQ27 is there a more recent edition?]

--Brown JQ, Srivastava R, Zhu H, McShane MJ. Enzymatic fluorescent microsphere glucose sensors:evaluation of response under dynamic conditions. Diabetes Technol Ther. 2006 Jun;8(3):288-295. doi:10.1089/ dia.2006.8.288 Medline

--Burge MR, Mitchell S, Sawyer A, Schade DS. Continuous glucose monitoring: the future of diabetes management. Diabetes Spectr. 2008:21(2):112-119. doi:10.2337/diaspect.21.2.112

--Caplin NJ, O'Leary P, Bulsara M, Davis EA, Jones TW. Subcutaneous glucose sensor values closely parallel blood glucose during insulin-induced hypoglycaemia. Diabet Med. 2003;20(3):238-241. doi:10.1046/j.1464$5491.2003 .00837 . x$

Cash KJ, Clark HA. Nanosensors and nanomaterials for monitoring glucose in diabetes. Trends Mol Med. 2010;16(12):584-593. https://doi. org/10.1016/j.molmed.2010.08.002

Clarke WL, Anderson S, Breton M, Patek S, Kashmer L, Kovatchev B. Closed-loop artificial pancreas using subcutaneous glucose sensing and insulin delivery and a model predictive control algorithm: the Virginia experience.J Diabetes Sci Technol. 2009;3(5):1031-1038. https://doi. org/10.1177/193229680900300506

See AQ3 in text Diabetes UK. Facts and figures. London: Diabetes UK; 2017

- Ebbesen M, Andersen S, Besenbacher F. Ethics in nanotechnology: starting from scratch? Bull Sci Technol Soc. 2006a Dec;26(6):451-462. doi:10.1177/0270467606295003

-- Ebbesen M, Jensen TG. Nanomedicine: techniques, potentials, and ethical implications. J Biomed Biotechnol. 2006b;2006(5):51516 Medline.

Eborall HC, Dallosso HM, McNicol S et al. Explaining engagement in selfmonitoring among participants of the DESMOND Self-monitoring Trial: a qualitative interview study. Fam Pract. 2015;32(5):596-602. https://doi. org/10.1093/fampra/cmv060

--Freitas RA Jr. What is nanomedicine? Nanomedicine. 2005; 1(1):2-9 doi:10.1016/j.nano.2004.11.003 Medline

Gerstein HC, Miller ME, Byington RP et al for the Action to Control Cardiovascular Risk in Diabetes Study Group. Effects of intensive glucose lowering in type 2 diabetes. N Engl J Med. 2008; 358(24):2545-2559. https://doi.org/10.1056/NEJMoa0802743

Guadagnini R, Halamoda Kenzoui B, Walker L et al. Toxicity screenings of nanomaterials: Challenges due to interference with assay processes and components of classic invitro tests. Nanotoxicology 2015; 9 Suppl 1:13-24. https://doi.org/10.3109/17435390.2013.829590

--Hase S, Kenyon C (2001) Moving from andragogy to heutagogy: implications for VE: Proceedings of Research to Reality: Putting VET Research to Work. Australian Vocational Education and Training Research Association (AVETRA), Adelaide, SA

Heinemann L. Finger pricking and pain: a never ending story J Diabetes Sci Technol. 2008; 2(5):919-921. https://doi. org/10.1177/193229680800200526

Heo YJ, Takeuchi S. Towards smart tattoos for continuous glucose monitoring. Adv Healthc Mater. 2013; 2(1):43-56. https://doi.org/10.1002/ adhm.201200167

Holman RR, Paul SK, Bethel MA, Neil HAW, Matthews DR. Longterm follow-up after tight control of blood pressure in type 2 diabetes. N Engl J Med. 2008; 359(15): 1565-1576. https://doi.org/10.1056/ NEJMoa0806359

Holt P. Blood glucose monitoring in diabetes. Nurs Stand. 2014; 28(27):52-58. https://doi.org/10.7748/ns2014.03.28.27.52.e650

Hughes MD. The business of self-monitoring of blood glucose: a market profile. J Diabetes Sci Technol. 2009; 3(5): 1219-1223. https://doi. org $/ 10.1177 / 193229680900300530$

International Diabetes Federation. IDF diabetes atlas. 8th edn. 2017. www. diabetesatlas.org. (accessed: 8 January 2019)

Juliano RL. The future of nanomedicine: promises and limitations. Science and Public Policy 2012;39(1): 99-104. https://doi.org/10.3152/0302342 2X13214603531969

Kelly K. The inevitable: understanding the 12 technological forces that will shape our future. New York, NY: Penguin Books; 2016

Kerr D. Poor numeracy: the elephant in the diabetes technology room. J Diabetes Sci Technol. 2010; 4(6):1284-1287. https://doi org/10.1177/193229681000400601

-- Klonoff DC. The benefits of implanted glucose sensors. J Diabetes Sci Technol. 2007 Nov;1(6):797-800. doi:10.1177/19322968070010060 Medline 
--Kroto HW, Heath JR, O'Brien SC, Curl RF, Smalley RE. C 6 Buckminsterfullerene. Nature. 1985;318(6042):162-163. https://doi. org $/ 10.1038 / 318162 \mathrm{a} 0$

-Kumar V, Cotran RS, Robbins SL. (2003) Physiology 7th edn. Saunders, London. [AQ28 is there a more recent edition?]

-Lewis C. Home is where the heart monitor is. Emerging trends in medical device technology. FDA Consum. 2001 May-Jun;35(3):10-15 Medline.

Long R, McShane M. Optical instrument design for interrogation of dermally-implanted luminescent microparticle sensors. Conf Proc IEEE Eng Med Biol Soc. 2008; 2008:5656-5659. https://doi.org/10.1109/ IEMBS.2008.4650497

Malysheva A, Lombi E,Voelcker NH. Bridging the divide between human and environmental nanotoxicology. Nat Nanotechnol 2015; 10(10):835-44. https://doi.org/10.1038/nnano.2015.224

Marchant G. Small is beautiful: what can nanotechnology do for personalized medicine? Current Pharmacogenomics and Personalized Medicine. 2009; 7(4):231-237. https://doi.org/10.2174/187569209790112346

Maynard AD. Nanotechnology: the next big thing, or much ado about nothing? Ann Occup Hyg. 2007; 51(1):1-12. https://doi.org/ 10.1093/ annhyg/mel071

Meetoo DD. Insulin: an evolving paradigm in diabetes management. British Journal of Healthcare Management. 2013;19 Sup1:8-15. https://doi. org/10.12968/bjhc.2013.19.Sup1.8

Meetoo D, Lappin M. Nanotechnology and the future of diabetes management. Journal of Diabetes Management. 2009; 13(8):288-297. http://usir.salford.ac.uk/13952 (accessed 8 January 2019)

--Mnyusiwalla A, Daar AS, Singer PA. Mind the gap: science and ethics in nanotechnology. Nanotechnology. 2003 Mar 01;14(3):R9-R13. doi:10.1088/0957-4484/14/3/201

Moorley C, Chinn T. () Developing nursing leadership in social media. J Adv Nurs. 2016; 72(3):514-520. https://doi.org/10.1111/jan.12870

-Morrison I. (2000) Health Care in the New Millennium:Vision, Values and Leadership. Jossey-Bass, New Jersey. [AQ29 is there a more recent edition?]

ou X, Lennartz MR, Loegering DJ, Stenken JA. Long-term calibration considerations during subcutaneous microdialysis sampling in mobile rats. Biomaterials. 2010; 31(16):4530-4539. https://doi.org/10.1016/j. biomaterials.2010.02.016

--Nathan DM, Genuth S, Lachin J et al for Diabetes Control and Complications Trial Research Group. The effect of intensive treatment of diabetes on the development and progression of long-term complications in insulin-dependent diabetes mellitus. N Engl J Med. 1993; 329(14):977986. doi:10.1056/NEJM199309303291401

National Institute for Health and Care Excellence. Type 2 diabetes in adults: management. [NG28]. 2015. www.nice.org.uk/guidance/ng28 (accessed 8 January 2019).

-National Technology Initiative. (2000) Leading to the next industrial revolution, National Science and Technology Council's Committee on Technology. Available at: http://clinton4.nara.gov/media/pdf/nni.pdf. Accessed on: 6 December 2010

--Oberdörster G, Sharp Z, AtudoreiV et al.Translocation of inhaled ultrafine particles to the brain. Inhal Toxicol. $2004 \mathrm{Jan} ; 16(6-7): 437-445$ doi:10.1080/08958370490439597 Medline

--Ohkubo Y, Kishikawa H, Araki E, Miyata T, Isami S, Motoyoshi S, Kojima Y, Furuyoshi N, Shichiri M. Intensive insulin therapy prevents the progression of diabetic microvascular complications in Japanese patients with noninsulin-dependent diabetes mellitus: a randomized prospective 6-year study Diabetes Res Clin Pract. 1995 May;28(2):103-117. doi:10.1016/01688227(95)01064-K Medline

Pickup JC, Zhi ZL, Khan F, Saxl T, Birch DJS. Nanomedicine and its potential in diabetes research and practice. Diabetes Metab Res Rev. 2008; 24(8):604-610. https://doi.org/10.1002/dmrr.893

Poolsup N, Suksomboon N, Rattanasookchit S. Meta-analysis of the benefits of self-monitoring of blood glucose on glycemic control in type 2 diabetes patients: an update. Diabetes Technol Ther. 2009; 11(12): 775-784. https://

\section{KEY POINTS}

- Diabetes mellitus is characterised by chronically elevated blood glucose levels

- Maintaining blood glucose within the recommended parameters is important to prevent or slow the development of micro and macrovascular complications

- The smart tattoo is a revolutionary technological advancement designed to continually monitor blood glucose levels in real time

- In revolutionising health care, nanomedicine will inevitably have an impact on nursing as a profession

- Nurses need to extend their knowledge of ethics to proactively debate the ethical dimension of nanotechnology

doi.org/10.1089/dia.2009.0091

Rodbard D. Evaluating quality of glycemic control: graphical displays of hypoand hyperglycemia, time in target range, and mean glucose. J Diabetes Sci Technol. 2015; 9(1):56-62. https://doi.org/10.1177/1932296814551046

Simon J, Gray A, Clarke P et al for Diabetes Glycaemic Education and Monitoring Trial Group. Cost effectiveness of self monitoring of blood glucose in patients with non-insulin treated type 2 diabetes: economic evaluation of data from the DiGEM trial. BMJ. 2008; 336(7654):11771180. https://doi.org/10.1136/bmj.39526.674873.BE

--Stein EW, Grant PS, Zhu H, McShane MJ. Microscale enzymatic optical biosensors using mass transport limiting nanofilms. 1. Fabrication and characterization using glucose as a model analyte. Anal Chem. 2007 Feb;79(4):1339-1348, doi:10.1021/ac061414z Medline

--Stephens JW. Self-monitoring of blood glucose by people with type 2 diabetes. Diabetes Voice 2014. 59(1): 47-51. [AQ30 can't find ref. Please provide URL]

-Thompson RE. Nanotechnology: science fiction? Or next challenge for the ethics committee? Physician Exec. 2007 May-Jun;33(3):64-66 Medline.

Tomky D, Tomky D, Cypress M, Dang D, Maryniuk M, Peyrot M, Mensing C. Aade Position Statement. Diabetes Educ. 2008; 34(3):445-449. https://doi. org/10.1177/0145721708316625

-Traynor K. FDA holds public meeting on nanotechnology. Am J Health Syst Pharm. 2006 Nov 15;63(22):2175-2177. doi:10.2146/news060023 Medline

-UK Prospective Diabetes Study Group. Intensive blood-glucose control with sulphonylureas or insulin compared with conventional treatment and risk of complications in patients with type 2 diabetes (UKPDS 33). Lancet. 1998 Sep;352(9131):837-853. doi:10.1016/S0140-6736(98)07019-6 Medline

[AQ31 OK? found 2009 edition] Ullman-Cullere M, Clark E, Aronson S. Implications of Genomics for Clinical Informatics. In: Liu L, Özsu MT eds. Encyclopedia of Database Systems. B oston, MA: Springer; 2009. https://doi.org/10.1007/978-0-387-39940-9

Vigersky RA. Escaping the haemoglobin A1c-centric world in evaluating diabetes mellitus interventions. J Diabetes Sci Technol. 2015; 9(5):11481151. https://doi/org/10.1177/1932296815576363

-Vlasses FR, Smeltzer CH. Toward a new future for healthcare and nursing practice. JONA: The Journal of Nursing Administration. 2007 Sep;37(9):375-380. doi:10.1097/01.NNA.0000285140.19000.f5 Medline

Wang J. Electrochemical glucose biosensors. Chem Rev. 2008; 108(2):814-825. https://doi/org/10.1021/cr068123

Whitesides GM. The 'right' size in nanobiotechnology. Nat Biotechnol. 2003

Oct;21(10):1161-1165. doi:10.1038/nbt872 Medline

\section{CPD reflective questions [AQ32: can you add 3/4 reflective questions to encourage readers to reflect on their practice. Here are examples:}

- What can you do to enhance the safety of patients in your own clinical setting?

- Are there particular features of your clinical setting that may contribute to your patient feeling unsafe?

- Considering the seven themes, which can you identify as being one on which you could further reflect and consider with your wider team? 